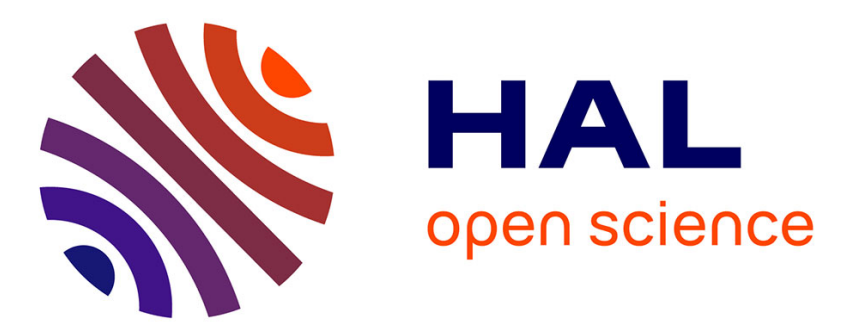

\title{
Conductive Filament Localization Within Crossbar Resistive Memories by Scanning Joule Expansion Microscopy
}

\author{
Etienne Puyoo, David Albertini
}

\section{To cite this version:}

Etienne Puyoo, David Albertini. Conductive Filament Localization Within Crossbar Resistive Memories by Scanning Joule Expansion Microscopy. IEEE Electron Device Letters, 2020, 41 (6), pp.848-851. 10.1109/LED.2020.2986543 . hal-03344468

\author{
HAL Id: hal-03344468 \\ https://hal.science/hal-03344468
}

Submitted on 15 Sep 2021

HAL is a multi-disciplinary open access archive for the deposit and dissemination of scientific research documents, whether they are published or not. The documents may come from teaching and research institutions in France or abroad, or from public or private research centers.
L'archive ouverte pluridisciplinaire $\mathbf{H A L}$, est destinée au dépôt et à la diffusion de documents scientifiques de niveau recherche, publiés ou non, émanant des établissements d'enseignement et de recherche français ou étrangers, des laboratoires publics ou privés. 


\title{
Conductive Filament Localization within Crossbar Resistive Memories by Scanning Joule Expansion Microscopy
}

\author{
Etienne Puyoo and David Albertini
}

\begin{abstract}
Control of filament growth within conductive bridge random access memories (CBRAM) is of crucial interest in order to ensure the reliability of such emerging devices. Here, we demonstrate that scanning joule expansion microscopy (SJEM) can be used to detect and precisely localize conductive filaments within operating crossbar CBRAM devices. Flexible memory devices based on $\mathrm{Pd} / \mathrm{Al}_{2} \mathrm{O}_{3} / \mathrm{Ag}$ stacks are first elaborated at low temperature on polyimide substrate. These devices show set and reset operations at low voltage $(<2 \mathrm{~V})$ with $\mathrm{ON} / \mathrm{OFF}$ ratios superior to $10^{4}$. Under operation in the low resistance state, the SJEM amplitude images reveal a hot spot underlying the presence of a single conductive filament. An effective thermal diffusion length of $4.3 \mu \mathrm{m}$ is extracted at $50 \mathrm{kHz}$ and it is also demonstrated that the thermal expansion signal is proportional to the dissipated Joule power. We believe that the proposed procedure opens the way to reliability studies that can be applied to any family of memory device based on filamentary conduction.
\end{abstract}

Index Terms - CBRAM, Flexible electronics, SJEM, Filament localization.

\section{INTRODUCTION}

In recent years, the thermal characterization of materials and devices at submicrometric scale has become a major issue regarding advances in the field of nanoelectronics. Scanning Joule Expansion Microscopy (SJEM) [1-3] is a technique based on atomic force microscopy (AFM) that already proved its efficiency to study thermal transport within nanoelectronic devices. In fact, SJEM is an imaging mode with a nanometric lateral resolution that detects surface displacements generated by local temperature variations within a device under test (DUT).

As examples presented in literature, SJEM has successfully been used to study thermal transport within thin metallic films [4] and carbon nanotubes devices [5], to characterize as well Joule and Peltier effects within phase change memory devices [6] and, more recently, to detect hot spots within threshold switching devices [7].

Here, we are using this imaging mode to detect and localize conductive filaments within conductive bridge random access memories (CBRAM) [8-10]. CBRAM is a class of non-volatile memory that stores the data as distinct resistance states. CBRAM is a two-terminal device generally made of two metal electrodes (one inert and the other one electrochemically active) with a thin solid electrolyte between them. The principle of CBRAM relies on redox reactions which enable the reversible formation/dissolution of metallic filaments between the two terminals. A low resistance state (LRS) is caused by the presence of at least one metallic bridge while the absence of filament results in a high resistance state (HRS). Understanding the mechanisms behind filament growth is of critical interest in order to improve the reliability of such emerging memories.

Several approaches based on transmission electron microscopy (TEM) have already been proposed in literature in order to visualize and precisely localize the nucleation sites of conductive filaments [11-12]. These different approaches are outstanding as they gave insight in the physical chemistry of metallic bridge formation. However, these approaches are destructive and use device configurations that are a bit far from the real crossbar architecture. Moreover, they do not give any information about heat dissipation and thermal expansion which most likely play a role in the filament formation and stabilization.

Other attempts based on AFM have also been proposed in literature to obtain filament's observation. In Ref. [13-14], the filament was located by scalpel microscopy which is also a destructive imaging technique. In this mode (scalpel microscopy), the AFM tip is used to etch the top electrode of the CBRAM device while subsequently Conductive-AFM (CAFM) mode is used to detect the filament. In Ref. [15], a conductive filament was observed by C-AFM but not on a real complete CBRAM device (a PtIr coated AFM tip was replacing the device top electrode). In Ref. [16], Scanning Thermal Microscopy (SThM) was used to analyze localized heating in a $\mathrm{MoTe}_{2}$-based memory. Although SThM is a nondestructive testing, MoTe2-based memories are not considered as CBRAM devices. Their principle of operation does not rely on the reversible formation/dissolution of a metallic bridge.

In this work, we demonstrate that SJEM can be used as a nondestructive surface displacement imaging technique in order to precisely detect the position of conductive bridges within crossbar CBRAM devices. Under operation in the LRS state, high current densities pass through the ultrathin conductive bridge generating heat dissipation and surface expansion that can be detected by monitoring the deflection of an AFM probe. 


\section{SJEM PRINCIPLE}

Figure 1 presents a schematic of the SJEM set-up. Surface expansion imaging was carried out with an AFM contact mode probe (Nanosensors PPP-CONT) mounted on a NTEGRA equipment from NTMDT. The Nanosensors PPP-CONT probe has a resonant frequency of $14 \mathrm{kHz}$ in a clamped-free

Fig. 1. SJEM principle.

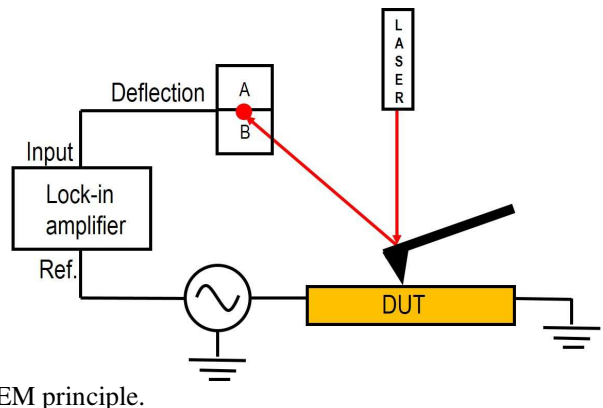

configuration in air. According to Ref. [18], when the probe is put in contact with a sample, its resonant frequency in a clamped-pinned configuration shifts to a value that is 4.39 times higher, namely around $60 \mathrm{kHz}$ here. All the experiments presented in the present paper were therefore carried out in contact mode with excitation frequencies in the range of 50$60 \mathrm{kHz}$ in order to optimize the sensitivity of the surface expansion measurement. As shown on Figure 1, the DUT was excited with a sine wave voltage generated by a HP 33120A waveform generator. The surface displacement image was obtained from a Signal Recovery DSP 7280 lock-in amplifier that analyses the deflection signal from the AFM quadrant photodiode.

It should be noted that the selected excitation frequency in the range of $50-60 \mathrm{kHz}$ was high enough to avoid any feedback control from the z-piezoelectric stage of the AFM equipment. The feedback loop bandwidth of the AFM controller is indeed close to $10 \mathrm{kHz}$. The sine wave excitation voltage $V(t)$ was composed of both DC and AC components, as given by the following expression:

$$
V(t)=V_{0}(1+\cos (2 \pi f t))
$$

where $V_{0}$ and $f$ are the excitation voltage amplitude and frequency, respectively.

The dissipated Joule power within the DUT can therefore be expressed as:

$$
P_{\text {Joule }}=\frac{V_{0}^{2}}{R}\left(\frac{3}{2}+2 \cos (2 \pi f t)+\frac{1}{2} \cos (4 \pi f t)\right)
$$

where $R$ is the total resistance of the DUT.

Here, we recall that the surface expansion signal purely originates from Joule dissipation. As a result, each frequency component of the Joule power $P_{\text {Joule }}$ induces a corresponding expansion signal component at the same frequency. From equation (2), we can see that the maximum peak power is dissipated at the $f$ frequency. We therefore decided to extract the first harmonic $f$ frequency deflection amplitude from the lock-in amplifier in order to construct the thermal expansion image of the operating CBRAM device.

\section{DUT DESCRIPTION}

As shown on Figure 2, the DUT is based on a $\mathrm{Pd} / \mathrm{Al}_{2} \mathrm{O}_{3} / \mathrm{Ag}$

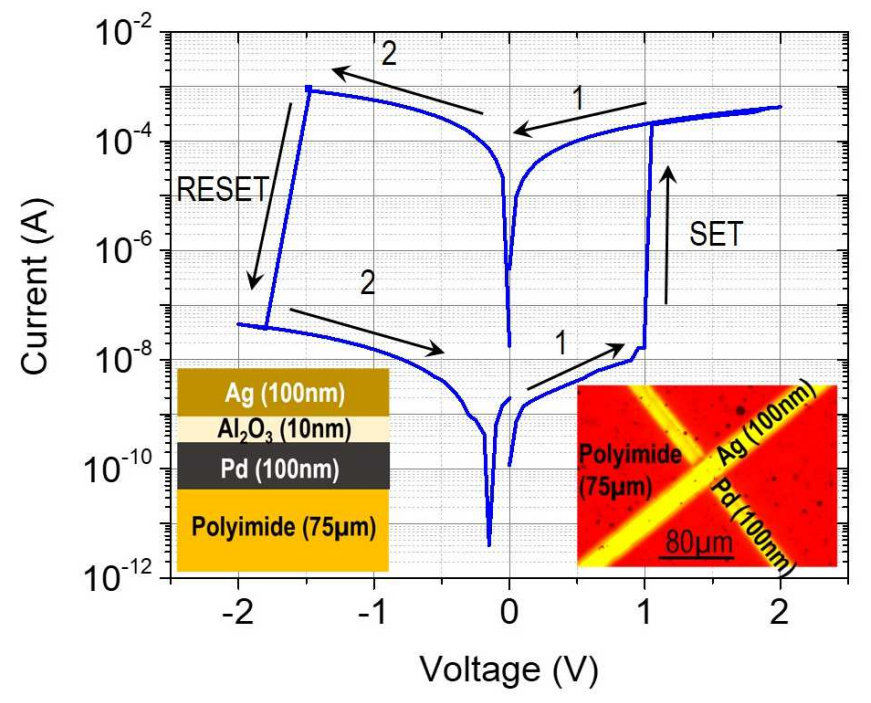

Fig. 2. Current-Voltage characteristic showing both SET and RESET operations. Insets: schematic of the CBRAM stack and optical microscope image of a CBRAM device.

stack integrated on a $75 \mu \mathrm{m}$ thick flexible polyimide substrate. The $\mathrm{Al}_{2} \mathrm{O}_{3}$ layer acts as a thin solid electrolyte while $\mathrm{Pd}$ and $\mathrm{Ag}$ electrodes respectively play the role of inert and electrochemically active electrodes. Both bottom Pd and top $\mathrm{Ag}$ electrodes were patterned by e-beam evaporation through solid shadow masks in a crossbar geometry and have a thickness of $100 \mathrm{~nm}$. The $10 \mathrm{~nm}$ thick $\mathrm{Al}_{2} \mathrm{O}_{3}$ solid electrolyte was grown by atomic layer deposition at $200^{\circ} \mathrm{C}$ from trimethyl-aluminum and $\mathrm{H}_{2} \mathrm{O}$ precursors (pulse and purge durations of $60 \mathrm{~ms}$ and $10 \mathrm{~s}$, respectively, for both precursors). The optical microscope image in the inset of Figure 2 shows a complete crossbar CBRAM device with a section of $30 \times 30 \mu \mathrm{m}^{2}$.

A typical current-voltage characteristic of the CBRAM device showing set and reset operations is also presented in Figure 2. The bottom Pd electrode was grounded while a voltage sweep was applied from the top Ag electrode. Under a positive voltage sweep from 0 to $2 \mathrm{~V}$, we can observe in Figure 2 that the CBRAM device switches from a HRS state to a LRS state at approximatively $1 \mathrm{~V}$. It should be noted that the two resistance states give rise to ON/OFF ratios superior to $10^{4}$. According to literature [11], this set operation is due to the oxidation of the Ag electrode and the subsequent migration of $\mathrm{Ag}^{+}$cations that generate a conductive metallic bridge within $\mathrm{Al}_{2} \mathrm{O}_{3}$ between top and bottom electrodes. Then, applying a negative voltage sweep from 0 to $-2 \mathrm{~V}$ reverses the growth of $\mathrm{Ag}$ filament and generates a reset operation at around $-1.5 \mathrm{~V}$ which corresponds to the filament break.

\section{RESULTS AND DISCUSSION}

Prior to perform thermal expansion imaging, the DUT was switched to its LRS state in order to generate a conductive Ag filament within the stack. The exciting sine wave voltage was then kept positive (with Pd bottom electrode grounded) in order to avoid any reset operation and filament breaking during SJEM imaging. 

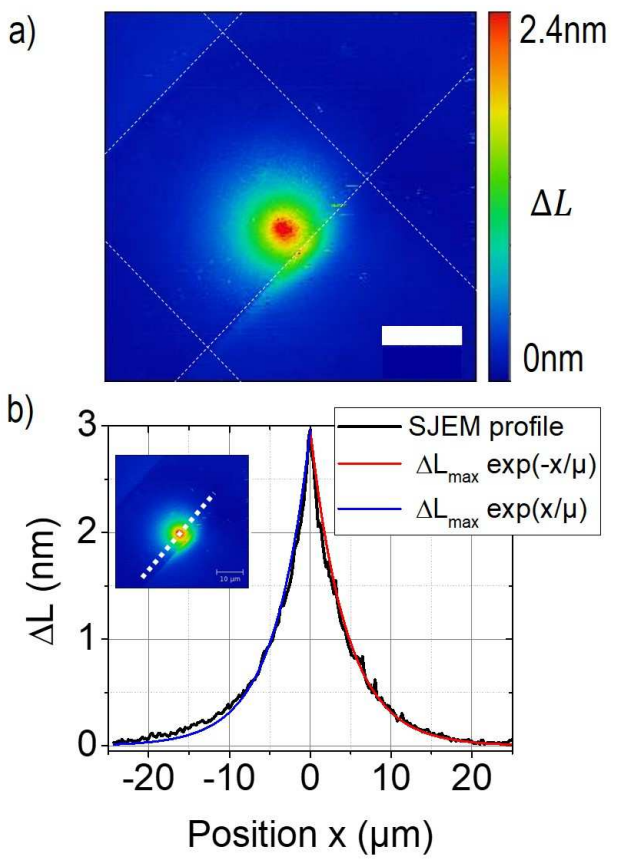

Fig. 3 a) Amplitude image of a CBRAM device submitted to a $1 \mathrm{Vpp}$ sine voltage (scale bar is $10 \mu \mathrm{m}$ ); b) SJEM profile centered on the hot spot revealing the presence of the conductive filament.

The total resistance of the CBRAM device in LRS state is $4850 \Omega$ while the $\mathrm{Pd}$ and $\mathrm{Ag}$ lines have electrical resistances both inferior to $10 \Omega$. In LRS state, the total resistance of the DUT corresponds to the combination in series of the Pd and Ag lines resistances with the resistance of the Ag conductive bridge. We can therefore consider that the main resistance of the DUT is coming from the Ag filament and that the majority of the power is dissipated within the $\mathrm{Ag}$ filament under operation.

Figure 3a presents a SJEM amplitude image of the DUT submitted to a $50 \mathrm{kHz}$ and $1 \mathrm{Vpp}$ sine wave voltage. The expansion signal was obtained by converting in nanometers the deflection signal from the quadrant photodiode of the AFM (initially expressed in volts). A conversion coefficient of $2193 \mathrm{~nm} / \mathrm{V}$ was obtained from the slope of a force curve performed directly on the DUT. A single hot spot corresponding to a maximum thermal expansion of $3 \mathrm{~nm}$ in the vertical direction is clearly visible close to an edge of the crossbar structure (Figure 3a). Note that the boarders of the bottom and top electrodes are indicated with superimposed white dashed lines on the SJEM image. The presence of this hot spot reveals the existence of a single Ag conductive filament within the CBRAM stack.

A SJEM profile centered on the observed hot spot is presented in Figure $3 \mathrm{~b}$ and is fitted by an exponential decay function given by the following expression:

$$
\Delta L=\Delta L_{\max } \exp (-|x| / \mu)
$$

where $\Delta L_{\max }$ is the maximum amplitude in the center of the hot spot, $x$ is the position relative to the center of the hotspot and $\mu$ is the effective thermal diffusion length of the DUT. A good agreement is found for $\Delta L_{\max }=3 \mathrm{~nm}$ and $\mu=4.3 \mu \mathrm{m}$.

Figure $4 \mathrm{a}$ presents a series of SJEM images performed at $60 \mathrm{kHz}$ with different excitation voltage amplitudes varying a)

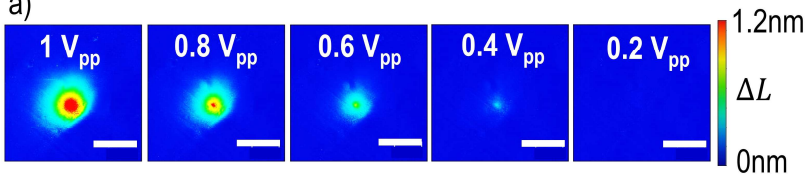

b)

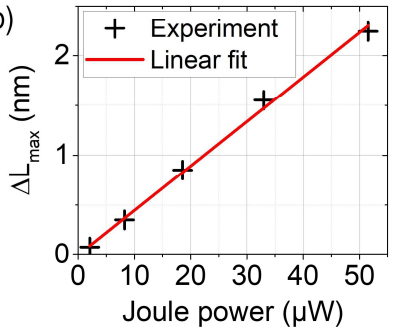

Fig. 4 a) SJEM images obtained for different excitation voltage amplitudes (scale bar is $15 \mu \mathrm{m}$ ); b) Maximum surface displacement as a function of Joule power.

from $0.2 \mathrm{Vpp}$ to $1 \mathrm{Vpp}$. The hot spot previously observed in Figure 3 is still present and shows a maximum surface displacement $\Delta L_{\max }$ which increases with respect to the excitation voltage amplitude. The maximum amplitude $\Delta L_{\text {max }}$ is plotted in Figure $4 \mathrm{~b}$ as a function of the Joule peak power $V_{0}{ }^{2} / R$. A clear linear relationship is observed in Figure $4 \mathrm{~b}$ which indicates that both the temperature variations and surface displacements of the DUT are proportional to the Joule peak power. This linear relationship is consistent with the results presented in $[2,3]$ and confirms that the SJEM signal is purely originated by Joule dissipation.

Future works will address the development of a thermomechanical model by Finite Element Modeling in order to perform spatially resolved quantitative thermometry of the memory device $[5,17]$.

\section{CONCLUSION}

In summary, we have proposed a procedure based on SJEM to detect and precisely localize a conductive filament within an operating crossbar CBRAM device. We have first elaborated flexible memory devices based on $\mathrm{Pd} / \mathrm{Al}_{2} \mathrm{O}_{3} / \mathrm{Ag}$ stacks at low temperature $\left(\leq 200^{\circ} \mathrm{C}\right)$ on flexible polyimide substrate. Such devices clearly showed set and reset operations at low voltage levels $(<2 \mathrm{~V})$ with ON/OFF ratios superior to $10^{4}$. Under excitation in the LRS state with a sine wave voltage, the SJEM amplitude image clearly exhibited a hot spot that revealed the presence of a stable Ag filament formed within the CBRAM stack. An effective thermal diffusion length of $4.3 \mu \mathrm{m}$ was extracted at a frequency of $50 \mathrm{kHz}$. It was also demonstrated that the thermal expansion signal was directly proportional to the Joule peak power. We believe that this non-destructive imaging mode opens the way to reliability studies that could be applied not only on CBRAM devices but to any family of resistive memory based on filamentary conduction.

\section{ACKNOWLEDGMENT}

The authors gratefully acknowledge D. Deleruyelle and C. Malhaire from INL for fruitful discussions. We also thank $\mathrm{J}$. Grégoire from the NanoLyon platform for its support and cooperation. 


\section{REFERENCES}

[1] J. Varesi and A. Majumdar, "Scanning Joule Expansion Microscopy at nanometer scales", Appl. Phys. Lett., vol. 72, pp.37-39, 1998. https://doi.org/10.1063/1.120638

[2] M. Cannaerts, D. Buntinx, A. Volodin and C. Van Haesendonck, "Calibration of scanning Joule microscope", Appl. Phys. A, vol. 72, pp. 67-70, 2001.

\section{https://doi.org/10.1007/s003390100648}

[3] S. Grauby, L.-D. Patino Lopez, A. Salhi, E. Puyoo, J.-M. Rampnoux, W. Claeys and S. Dilhaire, "Joule expansion imaging techniques on microelectronic devices", Microelectronics Journal, vol. 40, pp. 1367-1372, 2009.

https://doi.org/10.1016/j.mejo.2008.04.016

[4] S. P. Gurrum, W. P. King, Y. K. Joshi and K. Ramakrishna, "Size effect on the thermal conductivity of thin metallic films investigated by Scanning Joule Expansion Microscopy", Journal of heat transfer, vol. 130, pp. 082403, 2008. https://doi.org/10.1115/1.2928014

[5] X. Xie, K. L. Grosse, J. Song, C. Lu, S. Dunham, F. Du, A. E. Islam, Y. Li, Y. Zhang, E. Pop, Y. Huang, W. P. King and J. A. Rogers, "Quantitative thermal imaging of single-walled carbon nanotube devices by Scanning Joule Expansion Microscopy", ACS Nano, vol. 6, pp. 10267, 2012.

https://doi.org/10.1021/nn304083a

[6] K. L. Grosse, E. Pop and W. P. King, "Heterogeneous nanometer-scale Joule and Peltier effects in sub-25nm thin phase change memory devices", Journal of Applied Physics, vol. 116, pp. 124508, 2014.

https://doi.org/10.1063/1.4896492

[7] J. M. Goodwill, G. Ramer, D. Li, B. D. Hoskins, G. Pavlidis, J. J. Mc Clelland, A. Centrone, J. A. Bain and M. Skowronski, "Spontaneous current constriction in threshold switching devices", Nature Communications, vol. 10 , pp. 1628,2019

https://doi.org/10.1038/s41467-019-09679-9

[8] C. Gopalan, Y. Ma, T. Gallo, J. Wang, E. Runnion, J. Saenz, F. Koushan, P. Blanchard and S. Hollmer, "Demonstration of Conductive Bridging Random Access Memory (CBRAM) in logic CMOS process", Solid-State Electronics, vol. 58, pp. 54, 2011

https://doi.org/10.1016/j.sse.2010.11.024

[9] M. Zhang, S. Long, G. Wang, X. Xu, Y. Li, Q. Liu, H. Lv, X. Lian, E. Miranda, J. Sune and M. Liu, "Set statistics in conductive bridge random access memory device with $\mathrm{Cu} / \mathrm{HfO} 2 / \mathrm{Pt}$ structure", Appl. Phys. Lett., vol. 105, pp. $193501,2014$.

https://doi.org/10.1063/1.4901530

[10] Y.-T. Tseng, I.-C. Chen, T.-C. Chang, J.C. Huang, C.-C. Shih, H.-X. Zheng, W.-C. Chen, M.-H. Wang, W.-C. Huang, M.-C. Chen, X.-H. Ma, Y. Hao and Simon M. Sze, "Enhanced electrical behavior from the galvanic effect in Ag-Cu alloy electrode conductive bridging resistive switching memory", Appl. Phys. Lett., vol. 113, pp. 053501, 2018.

https://doi.org/10.1063/1.5023527

[11] Y. Yang, P. gao, S. Gaba, T. Chang, X. Pan and W. Lu, "Observation of conducting filament growth in nanoscale resistive memories", Nature Communications, vol. 3, pp. 732, 2012.

https://doi.org/10.1038/ncomms 1737

[12] W. A. Hubbard, A. Kerelsky, G. Jasmin, E. R. White, J. Lodico, M Mecklenburg and B. C. Regan, "Nanofilament formation and regeneration during $\mathrm{Cu} / \mathrm{Al}_{2} \mathrm{O}_{3}$ resistive memory switching", Nano Letters, vol. 15, pp. 3983, 2015.

https://doi.org/10.1021/acs.nanolett.5b00901

[13] U. Celano, L. Goux, K. Opsomer, M. Iapichino, A. Belmonte, A. Franquet, I. Hoflijk, C. Detavernier, M. Jurczak and W. Vandervost, "Scanning probe microscopy as a scalpel to probe filament formation in conductive bridging memory devices", Microelectronic Engineering, vol.120, pp. 67-70, 2014.

https://doi.org/10.1016/j.mee.2013.06.001

[14] U. Celano, L. Goux, A. Belmonte, K. Opsomer, A. Franquet, A. Schulze, C. Detavernier, O. Richard, H. Bender, M. Jurczak and W. Vandervost, "Threedimensional observation of the conductive filament in nanoscale resistive memory devices", Nano Lett., vol.14(5), pp. 2401-2406, 2014.

https://doi.org/10.1021/n1500049g

[15] D. Deleruyelle, M. Putero, T. Ouled-Khachroum, M. Bocquet, M.-V. Coulet, X. Boddaert, C. Calmes and C. Muller, " $\mathrm{Ge}_{2} \mathrm{Sb}_{2} \mathrm{Te}_{5}$ layer used as solid electrolyte in conductive-bridge memory devices fabricated on flexible substrate", Solid-State Electronics, vol. 79, pp. 159-165, 2013. https://doi.org/10.1016/j.sse.2012.06.010

[16] I. M. Datye, M. Munoz Rojo, E. Yalon, S. Deshmuhk, M. J. Mleczko and E. Pop, "Localized heating and switching in $\mathrm{MoTe}_{2}$-based resistive memory devices", Nano Lett., vol. 20(2), pp.1461-167, 2020.

https://doi.org/10.1021/acs.nanolett.9b05272

[17] E. Yalon, S. Deshmukh, M. Munoz Rojo, F. Lian, C. M. Neumann, F. Wiong and E. Pop, "Spatially resolved thermometry of resistive memory devices", Scientific Reports, vol. 7, pp. 15360, 2017. https://doi.org/10.1038/s41598-017-14498-3

[18] U. Rabe, "Atomic Force Acoustic Microscopy" in Applied Scanning Probe Methods II, Springer, pp. 37-90, 2006.

https://www.springer.com/gp/book/9783540262428 